\title{
Mitral Valve Disease, CTCAE
}

National Cancer Institute

\section{Source}

National Cancer Institute. Mitral Valve Disease, CT CAE. NCI Thesaurus. Code C143674.

A disorder characterized by a defect in mitral valve function or structure. 